\title{
Magnetic ordering and transitions of EuSe studied by x-ray diffraction
}

\author{
B. Díaz, ${ }^{1, *}$ E. Granado, ${ }^{2,3}$ E. Abramof, ${ }^{1}$ L. Torres, ${ }^{2,3}$ R. T. Lechner, ${ }^{4}$ G. Springholz, ${ }^{4}$ and G. Bauer ${ }^{4}$ \\ ${ }^{1}$ Laboratório Associado de Sensores e Materiais, Instituto Nacional de Pesquisas Espaciais, Caixa Postal 515, \\ 12227-010 São José dos Campos, SP, Brazil \\ ${ }^{2}$ Instituto de Física “Gleb Wataghin,” Universidade Estadual de Campinas (UNICAMP), Caixa Postal 6165, \\ 13083-970 Campinas, SP, Brazil \\ ${ }^{3}$ Laboratório Nacional de Luz, Síncrotron, Caixa Postal 6192, 13083-970 Campinas, SP, Brazil \\ ${ }^{4}$ Institut für Halbleiterphysik, Johannes Kepler Universität, A-4040 Linz, Austria
}

(Received 15 March 2010; revised manuscript received 25 April 2010; published 24 May 2010)

\begin{abstract}
The magnetic phase diagram and thermal expansion of EuSe is revisited using a high-quality 3200 - $\AA$-thick epitaxial film grown over a $(111) \mathrm{BaF}_{2}$ substrate. Resonant magnetic x-ray diffraction experiments reveal a highly hysteretic magnetic phase diagram between 1.8 and $T_{N} \sim 4.7 \mathrm{~K}$, in which two antiferromagnetic phases with propagation vectors $\vec{k}_{\mathrm{I}}=\left[\frac{1}{4}, \frac{1}{4},-\frac{1}{4}\right]$ (represented by $\uparrow \uparrow \downarrow \downarrow$ ) and $\vec{k}_{\mathrm{II}}=\left[\frac{1}{2}, \frac{1}{2},-\frac{1}{2}\right](\uparrow \downarrow \uparrow \downarrow)$ are observed. In addition, a defective phase with $\vec{k}_{\mathrm{III}}=[h, h,-h]\left(\frac{1}{4}<h<\frac{1}{3}\right)$ competes with the $\uparrow \uparrow \downarrow \downarrow$ phase. Details of the temperature dependence of $\vec{k}_{\text {III }}$ and corresponding peak widths indicate this phase is intermediate between $\uparrow \uparrow \downarrow \downarrow\left(h=\frac{1}{4}\right)$ and an ideal ferrimagnetic $\uparrow \uparrow \downarrow$ phase $\left(h=\frac{1}{3}\right)$, and may be represented by $(n \times \uparrow \uparrow \downarrow+\downarrow)$, with mean $n$ values between 7 and 2. High-resolution x-ray diffraction experiments show unit-cell hysteretic distortions that correlate with the transitions to the different magnetic phases existing below $T_{N}$.
\end{abstract}

DOI: 10.1103/PhysRevB.81.184428

PACS number(s): 75.70.-i, 75.50.Pp, 75.30.Kz, 61.05.cp

\section{INTRODUCTION}

Europium chalcogenides $\mathrm{Eu} X(X=\mathrm{O}, \mathrm{S}, \mathrm{Se}$, and $\mathrm{Te})$ are magnetic semiconductors with $\mathrm{NaCl}$ structure. Their magnetism results from the half-filled $4 f^{7}$ electronic shell of $\mathrm{Eu}^{2+}$ with $S=7 / 2$. The isotropic magnetic moments are effectively shielded by the $5 s^{2}$ and $5 p^{6}$ electronic shells, making the $\mathrm{Eu} X$ 's reference materials for Heisenberg magnetism. ${ }^{1}$

Studied since the sixties, the $\mathrm{Eu} X$ magnetic order is known to be ferromagnetic (FM) within individual (111), $(\overline{1} 11),(1 \overline{1} 1)$, or $(11 \overline{1})$ planes for each of the four distinct domains. Different magnetic orders are observed depending on whether successive FM planes order parallel ( $\uparrow)$ or antiparallel $(\uparrow \downarrow)$. This is determined by the ferromagnetic $J_{1}$ and antiferromagnetic (AFM) $J_{2}$ exchange constants among nearest and next-nearest neighbors, respectively. ${ }^{2}$ For EuO and EuS, $J_{1}>\left|J_{2}\right|$ results in ferromagnetic $\uparrow \uparrow \uparrow$ order. The opposite is true for EuTe, $J_{1}<\left|J_{2}\right|$, where $\uparrow \downarrow \uparrow \downarrow$ (or type-II) AFM order is observed. For EuSe, a delicate equilibrium, $J_{1} \sim\left|J_{2}\right|$, determines the existence of several magnetic phases, depending on temperature and history.

The interest on the $\mathrm{Eu} X$ family reappeared recently with the availability of high crystalline quality films grown by molecular-beam epitaxy (MBE). Photoluminescence and absorption lines were discovered with record-high $g$ factors with applied magnetic fields for EuTe and EuSe films. ${ }^{3-5}$ Also, magnetic correlations were observed among AFM EuTe layers through nonmagnetic PbTe spacers in EuTe/ PbTe superlattices. ${ }^{6}$

Though a great deal of experimental and theoretical ${ }^{7,8}$ efforts have been devoted to the $\operatorname{Eu} X$ family, their magnetic properties are far from being fully understood. Particularly for EuSe, several descriptions of its phase diagram can be found. In 1969, a neutron-diffraction study of EuSe by Fischer et al. ${ }^{9}$ identified a $\uparrow \uparrow \downarrow \downarrow$ (type-I) AFM state at $4.2 \mathrm{~K}$. At $1.9 \mathrm{~K}$ they observed coexistence of the $\uparrow \downarrow \uparrow \downarrow$ AFM and the $\uparrow \uparrow \downarrow$ ferrimagnetic phases. The three observed magnetic orderings, in which successive (111) planes order $\uparrow \uparrow \downarrow \downarrow$, $\uparrow \downarrow \uparrow \downarrow$, and $\uparrow \uparrow \downarrow$, are schematically represented in Fig. 1 . Griessen et al. ${ }^{10}$ used dilatometric and magnetic measurements to obtain a phase diagram of EuSe and determine the length changes during the magnetic transitions. They found that EuSe spin ordering becomes $\uparrow \uparrow \downarrow \downarrow$ below 4.6 K, $\uparrow \uparrow \downarrow$ below $2.8 \mathrm{~K}$, and $\uparrow \downarrow \uparrow \downarrow$ below $1.8 \mathrm{~K}$. They also found significant lattice contractions at $1.8 \mathrm{~K}$ and at $2.8 \mathrm{~K}$, and a small expansion at $4.6 \mathrm{~K}$, with increasing temperature.

A description of the hysteretic behavior of EuSe is first found in Callen and de Moura's work in 1977. ${ }^{11}$ According to them, cooling EuSe produces a first-order transition to the $\uparrow \uparrow \downarrow \downarrow$ phase at $4.6 \mathrm{~K}$, and below $2.8 \mathrm{~K}$ the $\uparrow \downarrow \uparrow \downarrow$ phase

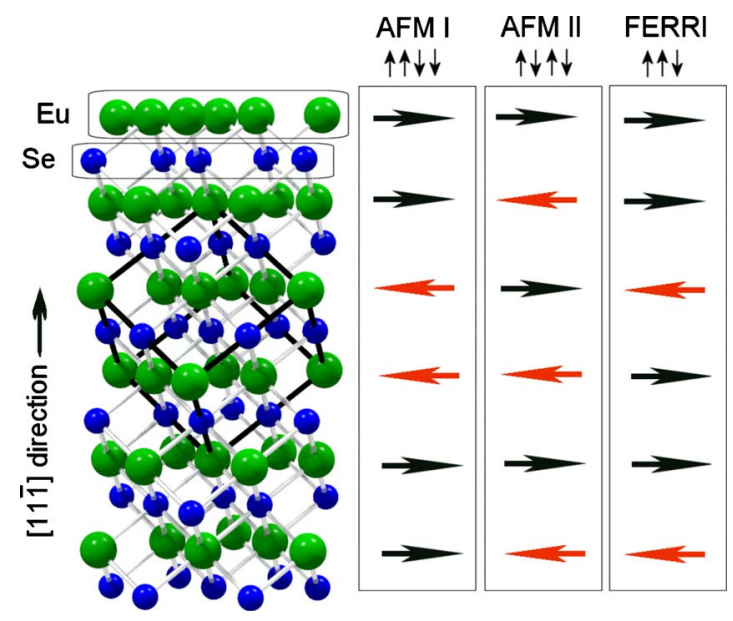

FIG. 1. (Color online) Structural and magnetic orderings of EuSe. To the left, the fcc lattice of EuSe is schematically represented, with the unit cell marked with darker bonds. To the right, the three observed magnetic orderings: AFM I, AFM II and ferrimagnetic, with different spin alignments in successive Eu atomic layers when viewed from a [111] equivalent direction. 
appears, coexisting with the $\uparrow \uparrow \downarrow \downarrow$ until below $1.9 \mathrm{~K}$. Heating back, the $\uparrow \uparrow \downarrow \downarrow$ phase transforms to $\uparrow \uparrow \downarrow$ at $1.9 \mathrm{~K}$, and then turns again into $\uparrow \uparrow \downarrow \downarrow$ at $\sim 4$ K. Existing $\uparrow \downarrow \uparrow \downarrow$ phase also turns into $\uparrow \uparrow \downarrow \downarrow$ at $\sim 2.5 \mathrm{~K}$. More recent experiments on EuSe films grown by $\mathrm{MBE}$ on $\mathrm{BaF}_{2}$ substrates, using superconducting quantum interference device (SQUID) magnetometry and magneto-optics Faraday and Kerr effects, did not find evidence the $\uparrow \uparrow \downarrow \downarrow$ phase, which was ascribed to the presence of strain in the epitaxial films. ${ }^{12}$ Overall, the scenario for the EuSe magnetic phases is still incomplete, and a detailed diffraction study of the magnetic phases of EuSe, including magnetoelastic changes and thermal hysteresis, is missing.

Magnetic resonant X-ray diffraction (MRXRD) is particularly suitable to study the magnetic structure of rare-earth compounds in the form of thin films. ${ }^{13}$ Our previous studies on EuTe films and EuTe/PbTe superlattices proved the suitability of MRXRD to study the magnetic orderings in this family, combining a strong resonance enhancement at Eu $L_{\mathrm{II}}$ and $\mathrm{Eu} L_{\mathrm{III}}$ edges, high surface sensitivity, and separation of the charge and magnetic signals through polarization analysis of the diffracted beam. ${ }^{14,15}$ In this work, we use MRXRD to study the magnetic order of an epitaxial EuSe film, grown by molecular-beam epitaxy on a (111) $\mathrm{BaF}_{2}$ substrate. This is complemented by high-resolution charge $\mathrm{x}$-ray diffraction to probe the magnetoelastic effects accompanying the magnetic phase transitions. Our results shed light onto the interesting magnetostructural phase diagram of EuSe. A detailed picture of the magnetic phases and their hysteretic behavior is given. The high resolution of MRXRD allows us to conclude that the ferrimagnetic phase usually identified as $\uparrow \uparrow \downarrow$ is actually a correlated-defect phase, intermediate between $\uparrow \uparrow \downarrow$ and $\uparrow \uparrow \downarrow \downarrow$ states.

\section{EXPERIMENTAL}

A $0.32 \mu \mathrm{m}$ EuSe film was grown in ultrahigh vacuum by molecular-beam epitaxy, on freshly cleaved (111) oriented $\mathrm{BaF}_{2}$ substrate, at $420{ }^{\circ} \mathrm{C}$. Eu and Se were evaporated from separate effusion cells with a flux ratio of 1:2. A polycrystalline $\mathrm{BaF}_{2}$ cap layer with $1000 \AA$ was grown on top of the EuSe layer at room temperature to protect it from oxidation.

For the initial structural characterization of the sample, high-resolution x-ray diffraction measurements were made, using a Philips X'Pert diffractometer in the triple axis configuration. A four crystals (220)Ge Bartels monochromator at the $\mathrm{Cu}$ x-ray tube output and a channel-cut $\mathrm{Ge}$ analyzer before the detector were employed during the triple axis measurements. X-ray magnetic resonant diffraction measurements were made at the XRD2 beamline of the Brazilian Synchrotron Laboratory (LNLS). ${ }^{16}$ The beamline is placed after a bending magnet, followed by a Rh-coated vertical focusing mirror, and a double-bounce Si(111) monochromator, in which the second crystal can be bent for sagittal focusing. The measurements were made at the Eu $L_{\mathrm{II}}$ absorption edge $(E=7614 \mathrm{eV}, \lambda=1.628 \AA)$, taking advantage of an enhancement of nearly 2 orders of magnitude in the magnetic scattered intensity. ${ }^{14} \mathrm{~A}$ polarimeter with a graphite analyzer was placed in the $2 \theta$ arm of the diffractometer during

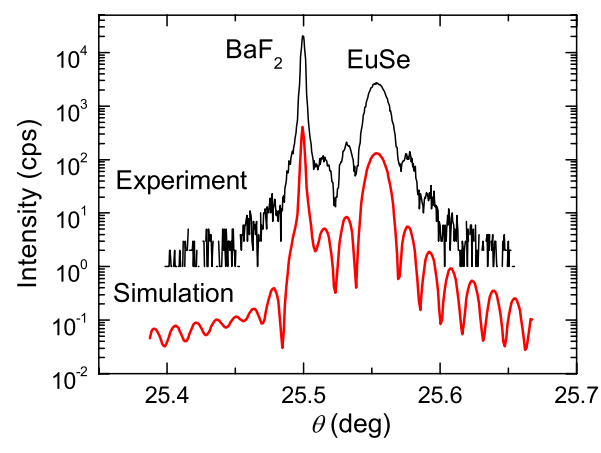

FIG. 2. (Color online) X-ray diffraction $\theta / 2 \theta$ scan along the HKL (222) reflections of the EuSe film and the $\mathrm{BaF}_{2}$ substrate at room temperature. Simulation of the diffracted spectra with dynamical diffraction theory of Takagi-Taupin (Refs. 17 and 18) considering an EuSe film fully strained to the substrate. The simulation was vertically shifted for better visualization.

the scattered beam polarization analysis, allowing to distinguish between $\sigma^{\prime}$ and $\pi^{\prime}$ scattered radiation. A Si(111) analyzer was used for the high-resolution magnetostriction measurements. A commercial closed-cycle cryostat with an additional Joule-Thomson open-cycle circuit was mounted on the $(4+2)$ Huber diffractometer cradle, reaching a base temperature of $1.8 \mathrm{~K}$. Considering the great sensitivity of the magnetic phases to temperature and thermal history in EuSe, the temperature stability was about $1 \mathrm{mK}$, and the maximum temperature overshoot was below $10 \mathrm{mK}$.

\section{RESULTS AND DISCUSSION}

\section{A. Room-temperature structural characterization}

Prior to the $\mathrm{x}$-ray resonant magnetic experiments, highresolution $\mathrm{x}$-ray charge diffraction was employed to get a detailed picture of the crystalline quality and strain state of the EuSe film. To this goal, scans were made around the $\mathrm{BaF}_{2}$ (222) reflection, and around other symmetric and asymmetric EuSe reflections. Figure 2 shows the $\theta / 2 \theta$ scan along the $[h h h]$ direction of the EuSe film and $\mathrm{BaF}_{2}$ substrate. Several thickness fringes can be observed, denoting the good crystalline quality and sharp interfaces of the EuSe film. Figure 2 also displays the simulation using TakagiTaupin dynamic diffraction theory. ${ }^{17,18}$ The best fit to the measurement was obtained considering an EuSe film of 315 $\mathrm{nm}$, fully strained to the $\mathrm{BaF}_{2}$ substrate, i.e., with an in-plane parameter equal to that of $\mathrm{BaF}_{2}(6.1988 \AA)$. The $\mathrm{BaF}_{2}$ cap layer was disregarded during the simulation since it is expected to be polycrystalline. The fully strained choice is supported by the very small lattice mismatch between bulk EuSe and $\mathrm{BaF}_{2}$, of only $0.11 \%$ at room temperature.

The strained state of the EuSe layer was further investigated measuring several HKL reflections with different offsets $\phi$ relative to the $[h h h]$ surface normal. Figure 3 shows the lattice parameters obtained from the $2 \theta$ peak positions of five reflections. Since the lattice parameters lie approximately in a straight line when plotted versus $\sin ^{2}(\phi)$, we conclude that the EuSe film has a constant strain through its depth, ${ }^{19}$ within our resolution. The EuSe film in-plane lattice 


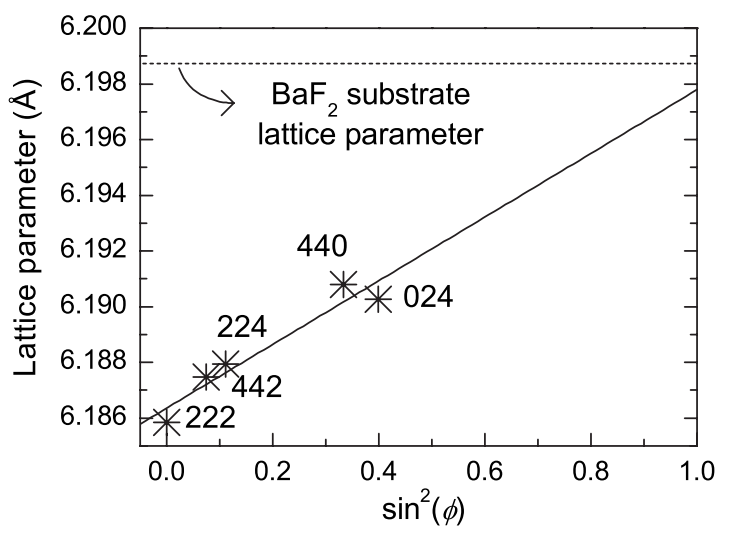

FIG. 3. Quasicubic lattice parameters calculated from different symmetric and asymmetric reflections of the EuSe film as a function of $\sin ^{2}(\phi)$, where $\phi$ is the reflection offset. The solid line is a linear fit to the experiment while the dashed horizontal line marks the $\mathrm{BaF}_{2}$ substrate lattice parameter.

parameter can be obtained from Fig. 3, extrapolating the linear behavior to $\sin ^{2}(\phi)=1$. The solid line in Fig. 3 is a linear fit, demonstrating that the in-plane parameter of the EuSe film nearly matches that of the $\mathrm{BaF}_{2}$ substrate. Considering the elastic constants of bulk EuSe, ${ }^{1}$ the EuSe film has a biaxial tensile strain of $0.10 \%$ and a perpendicular compressive strain of $-0.08 \%$. Therefore, strictly speaking our EuSe film shows a rhombohedral rather than cubic unit cell, in which the rhombohedral $\vec{c}$ axis points along the quasicubic [hhh] direction. Nonetheless, in order to allow for a direct comparison between our results and the previous descriptions of bulk $\mathrm{EuSe}$, we choose to represent the lattice parameters, Bragg reflections, and magnetic propagation vectors with respect to the quasicubic unit cell shown in Fig. 1.

\section{B. Magnetic phases}

Figure 4 shows a synchrotron x-ray diffraction reciprocalspace scan in the $[h h \bar{h}]$ direction, between (113) and (222) reflections at $3.2 \mathrm{~K}$, after cooling, at the Eu $L_{\mathrm{II}}$ edge. A background between $40-120$ counts per second (150 mA ring

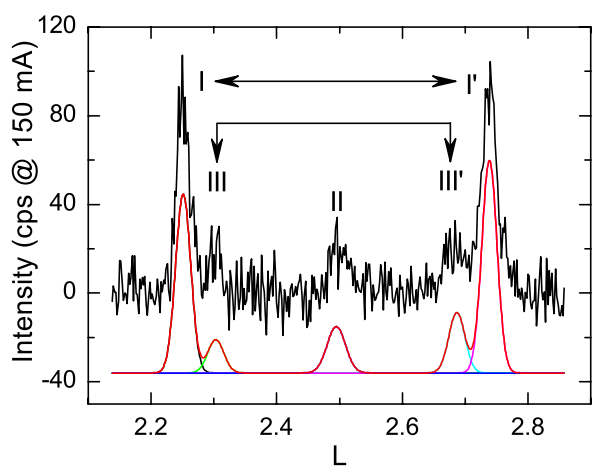

FIG. 4. (Color online) Scan between (113) and (222) HKL reflections. The measurement was made at $3.2 \mathrm{~K}$ (after cooling) and a scan at $10 \mathrm{~K}$ in the paramagnetic regime was subtracted. A fit to the experiment with five Gaussian peaks is also shown, shifted in the vertical for better visualization.

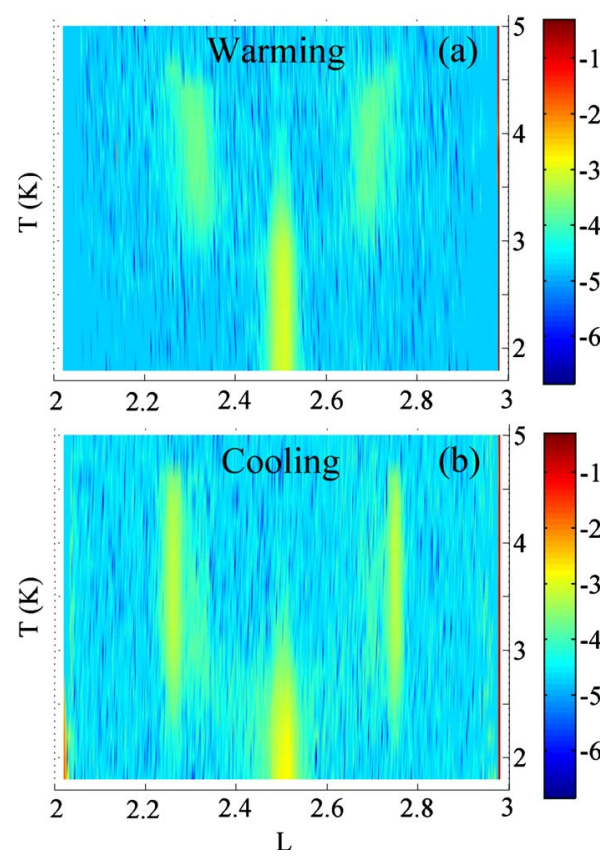

FIG. 5. (Color online) Magnetic diffraction along $[h h \bar{h}]$ direction, between the (113) and (222) HKL reflections, (a) warming from 1.8 to $5 \mathrm{~K}$, and (b) then cooling back to $1.8 \mathrm{~K}$.

current), measured in the paramagnetic state at $10 \mathrm{~K}$, was subtracted to highlight the magnetic scattering at $3.2 \mathrm{~K}$. Five peaks are observed in Fig. 4 scan. The analysis of the polarization of the scattered beam yielded a $\sigma \rightarrow \pi^{\prime}$ polarization change during the scattering process, strongly indicating the magnetic character of these peaks. Energy-dependent measurements of the $\left(\frac{3}{2}, \frac{3}{2}, \frac{5}{2}\right)$ reflection intensity showed a strong dipolar resonance, with a maximum at the Eu $L_{\mathrm{II}}$ edge (not shown), such as found for EuTe, ${ }^{14}$ further evidencing the magnetic nature of the observed signal. The intensities of the strongest magnetic peaks at resonance were about $10^{-6}$ times weaker than the charge peaks. The five observed magnetic peaks indicate coexistence of three magnetic phases at this temperature, since the pair marked by I and $\mathrm{I}^{\prime}$ in Fig. 4 are peaks related to the same phase, as well as pair III and III'.

To investigate the temperature dependencies of the magnetic peaks, we measured reciprocal space scans in the $[h h \bar{h}]$ direction between HKL (113) and (222), at temperatures ranging from 1.8 to $5 \mathrm{~K}$, on warming and cooling (Fig. 5). A background scan measured at $10 \mathrm{~K}$ was again subtracted from all scans. A marked hysteresis is easily noticed when comparing the scans measured during the warming and cooling cycles.

In order to study the magnetic phases and transitions in more detail, the individual scans in Fig. 5 were fitted with five Gaussian peaks, for which the width of peaks I and I' were constrained as equal, as well as those of peaks III and III' (Fig. 4). Also, the positions of peaks I, I' and III, III' were considered symmetric around the center of the scans at $\left(\frac{3}{2}, \frac{3}{2}, \frac{5}{2}\right)$. An example of such a fit is shown in Fig. 4 .

Figures 6(a)-6(f) shows the integrated intensity, propagation vector $\vec{k}=[h h \bar{h}]$, and peak widths $\Delta h$ of the observed magnetic phases as a function of temperature, under warm- 


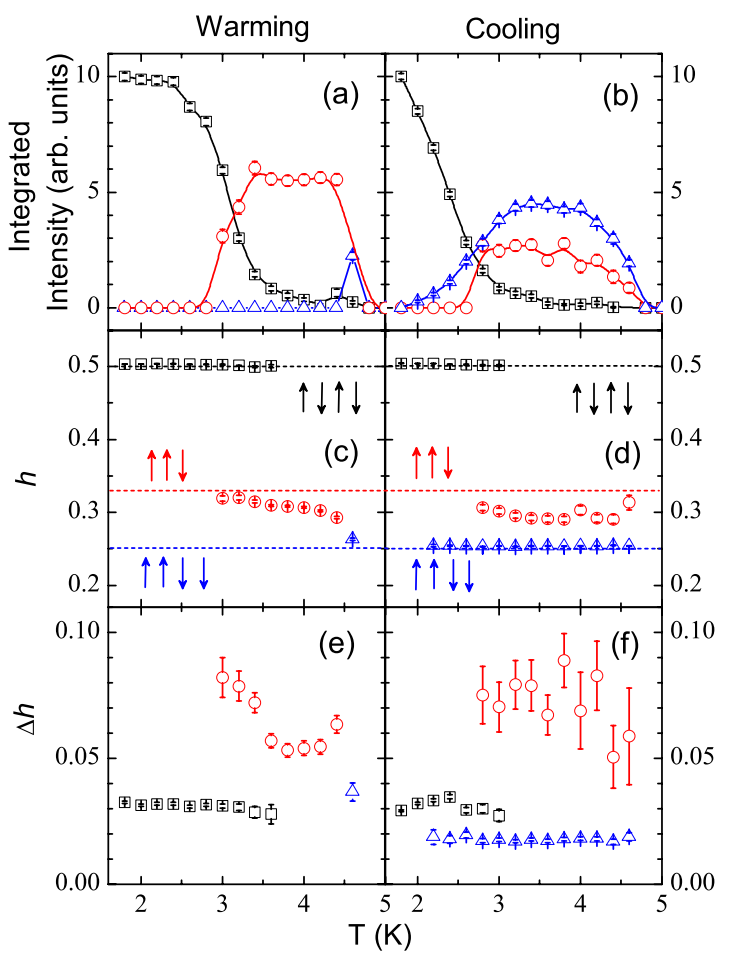

FIG. 6. (Color online) [(a) and (b)] Integrated intensity, [(c) and (d)] propagation vector $\vec{k}=[h h \bar{h}]$, and [(e) and (f)] peak widths $\Delta h$ of the observed magnetic phases under warming (left) and cooling (right). The dashed lines in (c) and (d) represents the expected $h$ values for $\uparrow \downarrow \uparrow \downarrow, \uparrow \uparrow \downarrow$, and $\uparrow \uparrow \downarrow \downarrow$ magnetic phases.

ing and cooling. Starting at $1.8 \mathrm{~K}$ [Fig. 6(a)], a single magnetic phase with $\vec{k}_{\mathrm{II}}=\left[\frac{1}{2}, \frac{1}{2},-\frac{1}{2}\right]$ is observed. The propagation vector of this phase remains locked for all temperatures in which it is observed [Fig. 6(c)], corresponding to the $\uparrow \downarrow \uparrow \downarrow$ phase identified in previous neutron-diffraction studies. ${ }^{11}$ As the sample is warmed above $\sim 3 \mathrm{~K}$, another phase appears, with an initial $\vec{k}_{\text {III }}$ close, but slightly different of $\left[\frac{1}{3}, \frac{1}{3},-\frac{1}{3}\right]$ at $3 \mathrm{~K}$ [Figs. 6(a) and 6(c)]. Clearly, this is the phase identified as ferrimagnetic $\uparrow \uparrow \downarrow$ in previous studies. The Bragg peaks for this phase are considerably broader than for $\uparrow \downarrow \uparrow \downarrow$ phase [Fig. 6(e)], indicating a highly defective and/or short-ranged phase. Above $\sim 3.5 \mathrm{~K}$, the $\uparrow \downarrow \uparrow \downarrow$ phase disappears. On further warming, the Bragg peaks of the defective phase become narrower and its propagation vector gradually moves, with a final jump to $\vec{k}_{\mathrm{I}} \sim\left[\frac{1}{4}, \frac{1}{4},-\frac{1}{4}\right]$ immediately below the paramagnetic Néel temperature, $T_{N} \sim 4.7 \mathrm{~K}$.

On cooling, a remarkably different equilibrium between the competing magnetic states is observed. Immediately below $T_{N}$, a coexistence between the defective phase and a phase with locked $\vec{k}_{\mathrm{I}} \sim\left[\frac{1}{4}, \frac{1}{4},-\frac{1}{4}\right]$ with sharp Bragg peaks (associated with the $\uparrow \uparrow \downarrow \downarrow$ phase ${ }^{11}$ ) sets in. The intensities of these phases show a similar trend on cooling, with an enhancement near $T_{N}$, and a weakening below $\sim 2.5 \mathrm{~K}$, when the $\uparrow \downarrow \uparrow \downarrow$ phase again rises and dominates.

Identical results with respect to those shown in Figs. 5 and 6 were obtained for scans along the $[h \bar{h} h]$ and $[\bar{h} h h]$ directions, which were taken after performing $120^{\circ}$ azimuthal rotations of the sample around the $[h h h]$ direction.

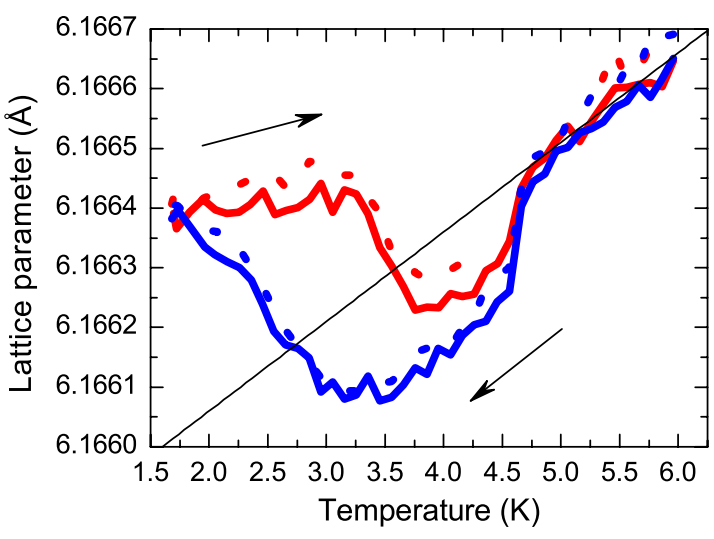

FIG. 7. (Color online) Temperature dependence of the lattice parameters obtained from the $2 \theta$ position of the (222) reflection during two successive (solid and dashed lines) cooling and heating cycles. The straight line is a linear fit to the paramagnetic region above $5 \mathrm{~K}$.

However, no magnetic peaks were observed at all in specular $\theta / 2 \theta$ scans along the $[h h h]$ normal direction.

\section{Magnetoelastic effects}

The high resolution achievable with synchrotron $\mathrm{x}$-ray diffraction is particularly useful to measure the subtle magnetoelastic distortions in the lattice that usually accompany magnetic transitions. To study the magnetostriction effects in the EuSe film, the incident beam was further collimated to lower the instrumental width and increase resolution. Also, a silicon analyzer [(111) reflection] was placed in front of the detector. With this optimized configuration, the position of the EuSe (222) HKL reflection was monitored as a function of temperature, in successive cooling and heating cycles. Figure 7 shows in-plane lattice parameter thus obtained as a function of temperature. Even though the variations are on the order of $10^{-4} \AA$, the behavior repeated through successive temperature cycles (solid and dashed lines).

In the paramagnetic region above $4.7 \mathrm{~K}$, the lattice parameter shows a linear increase due to thermal expansion (Fig. 7). The straight line in Fig. 7 is a linear fit to the data above $5 \mathrm{~K}$, yielding a thermal expansion coefficient of 15 $\times 10^{-6} \mathrm{~K}^{-1}$, similar to the value found in the literature $13.1 \times 10^{-6} \mathrm{~K}^{-1}$ at $300 \mathrm{~K} .{ }^{1}$ The deviation from this line below $4.7 \mathrm{~K}$ is due to magnetostriction effects. A clear hysteresis is observed when cycling the temperature in the cooling and warming directions. When cooling below $4.7 \mathrm{~K}$, a lattice contraction occurs rather abruptly, coincident with the first order transition to the $\uparrow \uparrow \downarrow \downarrow$ phase. Below $3 \mathrm{~K}$, the lattice parameter increases steadily down to $1.7 \mathrm{~K}$, clearly correlating with the emergence of the $\uparrow \downarrow \uparrow \downarrow$ phase [Figs. 5(a) and 6(b)]. Heating back, the lattice parameter is nearly constant while the $\uparrow \downarrow \uparrow \downarrow$ phase is dominant, up to $\sim 3.2 \mathrm{~K}$. Above this temperature, a lattice contraction takes place, coinciding to the emergence of the ferrimagnetic phase. On further warming, an expansion takes place at $\sim 4.7 \mathrm{~K}$, marking the transition to the paramagnetic phase.

\section{DISCUSSION}

Figures 5 and 6 show in detail the magnetic phases appearing at each temperature range, as well as the regions of 
coexistence, when cooling and warming the EuSe film. These results seem to contrast with those by Rumpf et al., ${ }^{12}$ who did not observe the $\uparrow \uparrow \downarrow \downarrow$ phase in a $2.5 \mu \mathrm{m}$ EuSe film, also grown by $\mathrm{MBE}$ on a $\mathrm{BaF}_{2}$ substrate. They used SQUID magnetometry and magneto-optics experiments to study the magnetic orderings, and the absence of the $\uparrow \uparrow \downarrow \downarrow$ phase in their sample was attributed to the presence of a residual strain in the epitaxial film. We emphasize that magnetic resonant x-ray diffraction is particularly suited for direct magnetic phase detection, and here it proves the existence of the $\uparrow \uparrow \downarrow \downarrow$ phase in a tensile strained EuSe film.

It is remarkable that the same magnetic peaks corresponding to the $\uparrow \downarrow \uparrow \downarrow, \uparrow \uparrow \downarrow \downarrow$, and defective phases are observed in all $[h h \bar{h}],[h \bar{h} h]$, and $[\bar{h} h h]$ directions, but not in the $[h h h]$ direction. These results demonstrate the existence of AFM domains with propagation vectors along the $[h h \bar{h}],[h \bar{h} h]$, and $[\bar{h} h h]$ oblique directions, but not along the specular $[h h h]$ direction. While all four directions are symmetry equivalent for bulk single crystals, this is not the case for the epitaxial film studied here, in which the symmetry is partially broken by the strain for the $[h h h]$ direction. This result is contrary to what occurs with EuTe films grown on (111) $\mathrm{BaF}_{2}$ substrates, where the $\uparrow \downarrow \uparrow \downarrow$ phase develops with propagation vector along the $[h h h]$ direction only. ${ }^{6}$ Opposite population of magnetic domains in EuTe and EuSe films grown on $\mathrm{BaF}_{2}$ can be explained by their respective strain state. Considering the lattice parameters of EuSe, EuTe, and $\mathrm{BaF}_{2}$ substrate, $6.192 \AA, 6.598 \AA$, and $6.199 \AA$, respectively, a biaxial tensile strain is expected for the EuSe film, while the EuTe film must be under biaxial compressive strain. The tensile strain in the EuSe film was confirmed above and compressive strains in EuTe films of $\sim-0.14 \%$ are still observed even for film thicknesses above $4 \mu \mathrm{m}$. Kepa et $a l .{ }^{6}$ demonstrated, using simple energy considerations, that compressive strain in EuTe favors in-plane domain population against oblique domain population. Compressive and tensile strains affect bonding lengths, and thus $J_{1}$ and $J_{2}$ exchange constants, opposite for in-plane and oblique domains, resulting in opposite domain population for EuTe and EuSe films.

The variety of magnetic phases observed in EuSe (Fig. 5) results from the close equilibrium among the nearestneighbors (NNs) and next-nearest-neighbors (NNNs) exchange constants $J_{1} \sim\left|J_{2}\right|$. With the same number of NN and NNN on adjacent (111) planes, the interplane exchange coupling, proportional to $J_{1}+J_{2}$, is very weak. Thus, other interactions such as dipolar, fourth-order, and magnetoelastic interactions become important to define the spin ordering. ${ }^{11,20,21}$ Which of these interactions is predominant is not yet well known.

Callen and de Moura ${ }^{11}$ suggested that magnetoelastic distortions played a fundamental role in the stabilization of the magnetic phases. The phase stabilization would occur as a result of the dependencies of $J_{1}$ and $J_{2}$ on interatomic distances, which have been widely investigated, both theoretically and experimentally. ${ }^{7,22-24}$ Our magnetoelastic studies show hysteretic lattice distortions that correlate well with all magnetic transitions when cycling the sample temperature. The $\uparrow \downarrow \uparrow \downarrow$ phase produces an expansion along the sample

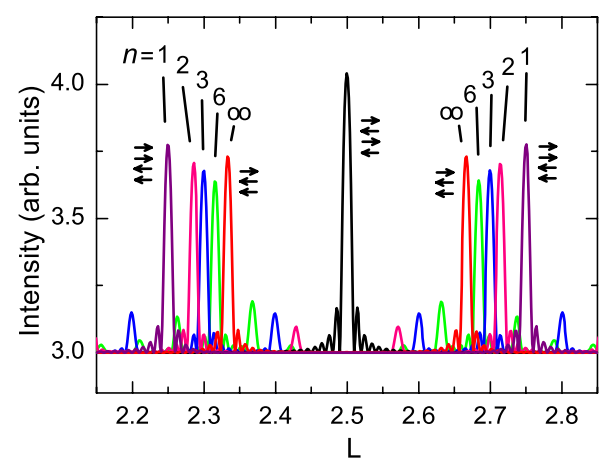

FIG. 8. (Color online) Kinematical simulation of the magnetic diffraction scan between (222) and (113) HKL. Peaks with $n$ $=2,3,6$ belong to correlated-defect phases with magnetic unit cells $[(n \times \uparrow \uparrow \downarrow)+\downarrow]$.

normal while $\uparrow \uparrow \downarrow$ and $\uparrow \uparrow \downarrow \downarrow$ phases produce contractions along the sample normal. However, the presence of multiple domains and of a biaxial strain on our EuSe film hinders a detailed study correlating the exchange constants and the lattice distortions measured, for which an unstrained singledomain sample would be ideal.

In order to understand the defective phase peaks position and drift, we performed kinematic simulations of the magnetic diffraction with different magnetic orderings. For this, we followed the approach outlined by Kepa et al., ${ }^{6}$ where the amplitudes scattered from successive FM (111) planes are summed up,

$$
\psi \propto \sum M_{j} \exp \left({ }_{l} Q_{z} j d\right)
$$

where $d$ is the spacing between magnetic Eu (111) planes, $Q_{z}$ is the scattering vector $(4 \pi / \lambda) \sin (\theta), \lambda$ is the wavelength, and $M_{j}$ is either 1 or -1 for $\uparrow$ or $\downarrow$ oriented planes, respectively. Figure 8 shows the results of these calculations for six different magnetic orientation sequences $M_{j}$. Three of the sequences represent the perfect AFM type-II $(\uparrow \downarrow \uparrow \downarrow \ldots)$, AFM type-I $(\uparrow \uparrow \downarrow \downarrow \ldots)$, and ferrimagnetic $(\uparrow \uparrow \downarrow \ldots)$ phases, while the other three sequences are ferrimagnetic phases with defects of different periodicity (Fig. 8). The defect phase unit cells consist of $n$ ferrimagnetic unit cells $\uparrow \uparrow \downarrow$ followed by an $\downarrow$ plane. In this way, defect phases are transitional between ferrimagnetic and AFM type-I phases, since for $n=1$ the defect phase reduces to AFM type-I $\uparrow \uparrow \downarrow \downarrow$, and for $n \rightarrow \infty$ it reduces to the ferrimagnetic $\uparrow \uparrow \downarrow$ phase.

The diffraction from the phases with periodic, correlated defects, yields peaks in between those of the ferrimagnetic and AFM type-I phases (Fig. 8). The peaks shift toward those of the AFM type-I phase as the defect periodicity increases (as $n$ decreases). This matches our experimental results shown in Figs. 5(a) and 6(c), where the peaks from the defective phase between 3 and $4.5 \mathrm{~K}$ shift to lower $h$ as temperature increases. The position of the experimental peaks varying from $h=0.32$ to 0.29 during warming [Fig. 6(c)] indicates defect-phase unit cells with $n=7$ to 2 . The considerable width of the defective phase peaks suggests a certain spread on the correlated defects wavelength at each temperature, likely due to tiny strain variations affecting the delicate 
equilibrium in EuSe film. The decrease in the defective phase peaks width as temperature rises [Fig. 6(e)] can be explained by a reduction in the spread on $n$ values.

Notice that the defect phase with $n=2$ has the highest possible defect concentration, becoming AFM type-I for $n$ $=1$. Hence, after a relative smooth shift to lower $h$ when $n$ goes from 7 to 2 , the correlated-defect model predicts a jump to lower $h$ for $n=1$, as shown in Fig. 8. This jump is observed in the experiments, during warming from 4.5 to $4.6 \mathrm{~K}$ [Figs. 5(a) and 6(c)]. The steep decrease on $h$ is accompanied by a sensitive reduction in the peak width [Fig. $6(\mathrm{e})]$, indicating longer range order.

Considering the similarities among the experimental and simulated magnetic peaks, we propose that the defective phase observed here, previously identified as $\uparrow \uparrow \downarrow$, is actually a correlated-defect phase with unit cell $[(n \times \uparrow \uparrow \downarrow)+\downarrow]$. The introduction of these defects should be energetically favorable since it lowers the overall magnetic moment of the $\uparrow \uparrow \downarrow$ phase. This correlated-defect phase is an intermediate case between $\uparrow \uparrow \downarrow$ and $\uparrow \uparrow \downarrow \downarrow$ phases, and results directly from the close equilibrium $J_{1} \sim\left|J_{2}\right|$ existing in EuSe, where the energy difference that stabilizes the various magnetic phases is very small.

\section{CONCLUSIONS}

The magnetic orderings of an EuSe film were determined using magnetic x-ray diffraction, particularly well suited for studies of magnetic phase equilibria. Profiting from the strong resonance enhancement observed at the Eu $L_{\mathrm{II}}$ absorption edge, we measured the magnetic Bragg peaks in successive cooling and warming cycles. A structural reflection was also measured in similar temperature cycles to study the magnetoelastic effects accompanying phase transitions. Three distinct magnetic phases were observed below $4.7 \mathrm{~K}$ : $\uparrow \uparrow \downarrow \downarrow, \uparrow \downarrow \uparrow \downarrow$, and a defective phase with $\vec{k}_{\mathrm{III}} \sim\left[\frac{1}{3}, \frac{1}{3},-\frac{1}{3}\right]$. Biaxial tensile strain kept the in-plane magnetic domain from being populated while the other three oblique domains were equally populated. A detailed picture of the magnetic phases temperature behavior, coexistence intervals, and hysteresis effects was given. The magnetic transitions were closely related to the magnetoelastic deformations of the EuSe film, with the $\uparrow \downarrow \uparrow \downarrow$ phase producing a lattice expansion, and the $\uparrow \uparrow \downarrow \downarrow$ and defective phases producing lattice contractions, all along the $[h h h]$ normal direction. Comparison with kinematic $x$-ray diffraction simulations suggest that the defective phase has unit cell $[(n \times \uparrow \uparrow \downarrow)+\downarrow]$. Values of $n$ from 7 to 2 satisfactorily reproduce the experimental peak positions and shifts during the warming of the sample.

\section{ACKNOWLEDGMENTS}

This work was partially supported by FAPESP and CNPq, Brazil.
*Corresponding author. Present address: Laboratório Nacional de Luz Síncrotron, Caixa Postal 6192, 13083-970 Campinas, SP, Brazil. beatriz.moreno@lnls.br

${ }^{1} \mathrm{P}$. Wachter, Handbook on the Physics and Chemistry of Rare Earths: Alloys and Intermetallics (North-Holland, Amsterdam, 1979), Vol. 2.

${ }^{2}$ T. Kasuya, J. Alloys Compd. 192, 11 (1993).

${ }^{3}$ W. Heiss, G. Prechtl, and G. Springholz, Phys. Rev. B 63, 165323 (2001).

${ }^{4}$ A. B. Henriques, A. Wierts, M. A. Manfrini, G. Springholz, P. H. O. Rappl, E. Abramof, and A. Y. Ueta, Phys. Rev. B 72, 155337 (2005).

${ }^{5}$ R. Kirchschlager, W. Heiss, R. T. Lechner, G. Bauer, and G. Springholz, Appl. Phys. Lett. 85, 67 (2004).

${ }^{6}$ H. Kępa, G. Springholz, T. M. Giebultowicz, K. I. Goldman, C. F. Majkrzak, P. Kacman, J. Blinowski, S. Holl, H. Krenn, and G. Bauer, Phys. Rev. B 68, 024419 (2003).

${ }^{7}$ J. Kunes, W. Ku, and W. E. Pickett, J. Phys. Soc. Jpn. 74, 1408 (2005)

${ }^{8}$ S. Q. Shi, C. Y. Ouyang, Q. Fang, J. Q. Shen, W. H. Tang, and C. R. Li, EPL 83, 69001 (2008).

${ }^{9}$ P. Fischer, W. Halg, W. von Wartburg, P. Schwob, and O. Vogt, Phys. Kondens. Mater. 9, 249 (1969).

${ }^{10}$ R. Griessen, M. Landolt, and H. R. Ott, Solid State Commun. 9, 2219 (1971).

${ }^{11}$ H. Callen and M. A. de Moura, Phys. Rev. B 16, 4121 (1977).
${ }^{12}$ K. Rumpf, P. Granitzer, W. Kellner, R. Kirchschlager, S. Janecek, H. Pascher, and H. Krenn, Acta Phys. Pol. A 105, 621 (2004).

${ }^{13}$ S. W. Lovesey and S. P. Collins, X-Ray Scattering and Absorption by Magnetic Materials (Oxford University Press, New York, 1996).

${ }^{14}$ B. Díaz, E. Granado, E. Abramof, P. H. O. Rappl, V. A. Chitta, and A. B. Henriques, Phys. Rev. B 78, 134423 (2008).

${ }^{15}$ B. Díaz, E. Granado, E. Abramof, P. H. O. Rappl, V. A. Chitta, and A. B. Henriques, Appl. Phys. Lett. 92, 242511 (2008).

${ }^{16}$ C. Giles, F. Yokaichiya, S. W. Kycia, L. C. Sampaio, D. C. Ardiles-Saravia, M. K. K. Franco, and R. T. Neuenschwander, J. Synchrotron Radiat. 10, 430 (2003).

${ }^{17}$ S. Takagi, J. Phys. Soc. Jpn. 26, 1239 (1969).

${ }^{18}$ D. Taupin, Bull. Soc. Fr. Mineral. Cristallogr. 87, 469 (1964).

${ }^{19}$ A. Pesek, Ph.D. thesis, Johannes Kepler Universität, Linz, 1993.

${ }^{20}$ K. Hiraoka, T. Hihara, K. Kojima, and T. Kino, Solid State Commun. 52, 479 (1984).

${ }^{21}$ H. Fukuma, T. Komatsubara, T. Suzuki, S. Kunii, E. Kaldis, and T. Kasuya, J. Magn. Magn. Mater. 31-34, 431 (1983).

${ }^{22}$ I. N. Goncharenko and I. Mirebeau, Phys. Rev. Lett. 80, 1082 (1998).

${ }^{23}$ K. Westerholt, A. Eiling, and H. Bach, J. Magn. Magn. Mater. 28, 214 (1982).

${ }^{24}$ K. Westerholt and H. Bach, Phys. Rev. B 31, 7151 (1985). 